\title{
Determinants of disease expression and survival of infected individual fronds in wild populations of Mazzaella laminarioides (Rhodophyta) in central and southern Chile
}

\author{
Alejandro H. Buschmann1, Juan A. Correa ${ }^{2, *}$, Jessica Beltrán ${ }^{2}$, Claudia A. Retamales ${ }^{1}$ \\ 'Departamento de Acuicultura, Universidad de Los Lagos, Casilla 933, Osorno, Chile \\ ${ }^{2}$ Departamento de Ecología, Facultad de Ciencias Biológicas, Pontificia Universidad Católica de Chile, Casilla 114-D, \\ Santiago, Chile
}

\begin{abstract}
This study provides evidence that the expression of a deformative disease in wild populations of the red algal host Mazzaella laminarioides is strongly influenced by exogenous factors. This was particularly the case for a population in Matanzas, Chile, whose members developed the symptoms when they grew in a high-infection zone, regardless of their history of susceptibility to the disease. However, our results for between Jocality transplants suggost that a genetic component may be playing a role in the expression of the disease. Plants transplanted from one locality to another did not reach the same levels of disease expression as their indigenous local counterparts. Infectious diseases seem to affect the survival of the infected hosts, an effect that varies depending on the pathogen involved, the season and the locality under consideration. In addition to the direct impacts of the 2 diseases (green patch disease caused by Endophyton ramosum and the deformative disease associated with Pleurocapsa sp.) affecting Mazzaella laminarioides, the deformative disease caused an increased susceptibility to grazing, which may accelerate the direct negative impact of the pathogen itself.
\end{abstract}

KEY WORDS: Endophytes Mazzaella - Transplant experiment Survivorship - Herbivore preference . Chile

\section{INTRODUCTION}

In recent years there has been a number of studies describing infections of different species of seaweeds by various endophytic pathogens (Correa et al. 1993, 1994, Correa \& McLachlan 1994, Weinberger et al. 1994, Correa \& Flores 1995). Most of these and previous studies (see Correa 1997 for a review) have concentrated on the morphological aspects of the associations. However, in laboratory experiments, Correa \& McLachlan (1992) demonstrated a negative effect of infections by endophytic green algae on growth, regeneration, carrageenan yield and toughness of the rhodo-

- Addressee for correspondence.

E-mail: jcorrea@genes.bio.puc.cl phycean host Chondrus crispus Stackh. The possibility of indirect, additional negative effects due to infection was also examined by these authors, who found that diseased host thalli were more attractive and more palatable to crustacean grazers (Correa \& McLachlan 1992). No equivalent study exists for any other infectious algal disease. From an ecological perspective, epidemiological studies have shown that, in spite of seasonal, latitudinal and spatial fluctuations, infection prevalence may affect an important fraction of a host population (Correa \& Sánchez 1996, Correa et al. 1997). Even though this information strongly suggests that pathogens may play an important role as a direct and indirect source of algal mortality and could modify the competitive abilities of diseased hosts in the field, no direct evidence exists to support this hypothesis. 
Mazzaella laminarioides (Bory) Fredericq is a perennial carrageenophytic alga dominating the mid and low intertidal zone of the wave-exposed coast in central and southern Chile (Santelices 1991). This alga is affected by endophytic organisms which are the cause of 2 diseases. The most common is the green patch disease caused by Endophyton ramosum Gardner (Correa et al. 1994), and the other is the deformative disease associated with infections by the cyanobacterium Pleurocapsa sp. (Correa et al. 1993). Our group has reported on several aspects (symptom characterization, host specificity, epidemiology, etc.) resulting from the interactions between $M$. laminarioides and its common endophytic pathogens (Correa et al. 1993. 1994, 1997. Correa \& Sánchez 1996, Sánchez et al. 1996). These studies revealed that fronds affected by the deformative disease had a patchy distribution within the host population, a phenomenon which persisted from year to year and within different populations. Theoretically, a clear spatial patchy distribution could result from particular micro-environments enhancing the expression of the disease or from patchy distribution of susceptible hosts. That is, the discase reaches a maximum expression in areas of the shore where susceptible individuals are available.

Taking into consideration the above information, this study was conceived to assess whether the factors potentially involved in the expression of the deformative disease are exogenous, endogenous, or both, by using transplant experiments within and between geo- graphically distant localities. This study also assessed the effects of the green patch disease and the deformative disease on host survival and tested the validity of generalizations regarding interactions between grazers and diseased hosts (Correa \& McLachlan 1992). In this context, evaluation of grazing-related effects secondary to pathogenic infections included feeding assays with crustacean and gastropod grazers.

\section{MATERIAL AND METHODS}

Disease expression. The localities selected for this study were Matanzas and Pucatrihue, Chile (Fig. 1a), which have been fully described elsewhere (Correa et al. 1997). Based on the patchy distribution of the deformative disease in these localities (Correa et al. 1997), areas with persistently high levels of fronds displaying full disease expression (high-infection zones) and others with a near absence of visually detectable diseased thalli (low-infection zones) were identified and selected for transplant experiments. Transplant units consisted of rock fragments, ca $9 \mathrm{~cm}^{2}$ each, containing abundant ( $\mathrm{Ca} 5$ to 20 ) each Mazzaella laminarioides fronds $\geq 2 \mathrm{~cm}$. These fragments were chipped off large granite boulders or platforms in the low-or highinfection zones using a chisel and a $0.5 \mathrm{~kg}$ hammer Fragments were subsequently glued to the rocks in the appropriate transplant locality by submarine epoxy cement (Stierling)

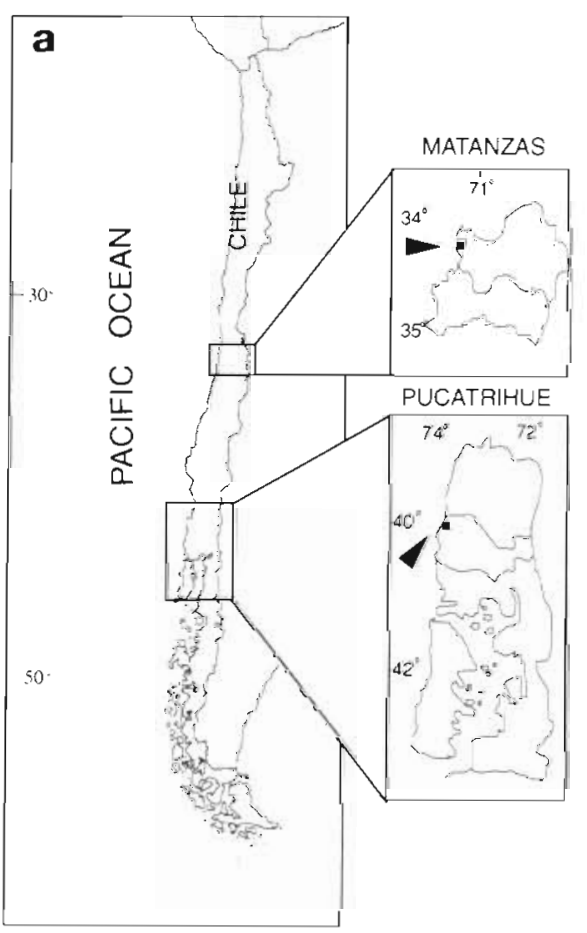

b

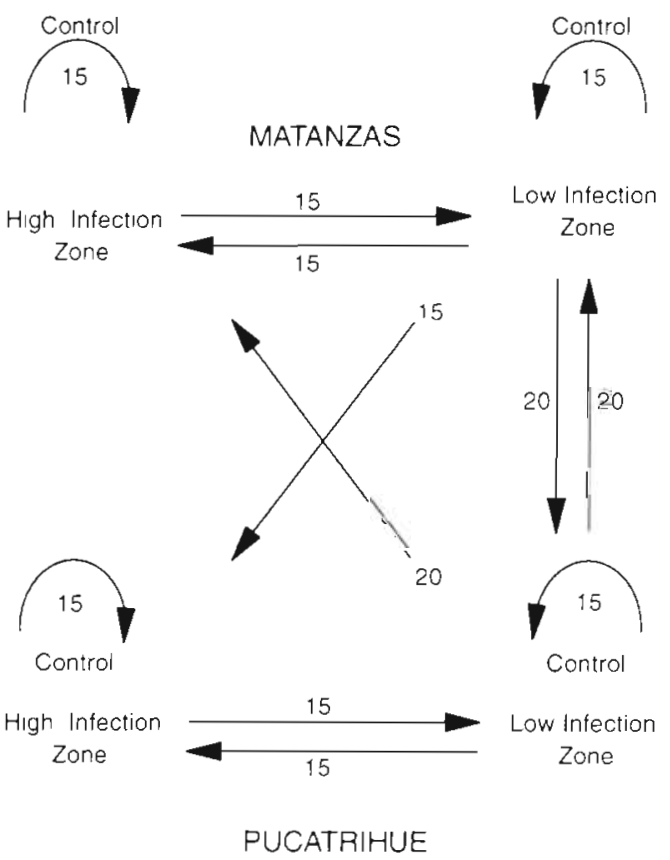

Fig. 1 (a) Localities in central and southern Chile and (b) scheme indicating origin and destination of Mazzaella laminarioides transplant units. Numbers indicate replicates used in each transplant 
Fig. 1b summarizes the origin and destination of the fronds during the transplant experiments. In Matanzas, a total of 15 fragments were transplanted from the low-infection zone to the high-infection zone and 15 others in the opposite direction. An identical protocol was followed in Pucatrihue. Additional fragments, 15 in each zone, were maintained in the 2 localities as controls for the experimental manipulations and to quantify the background level of infection. Interlocality transplants were done in addition to withinlocality transplants. In this case, 15 fragments from the low-infection zone in Matanzas were taken to Pucatrihue (high-infection zone) and 20 fragments collected at low-infection zones in Pucatrihue were taken to Matanzas (high-infection zone). Reciprocal interlocality transplants also included fragments from the low-infection zone in Matanzas to the low-infection zone at Pucatrihue, and vice versa. The whole experimental procedure, from obtaining the transplant units to the first data recording, was completed in a $3 \mathrm{~d}$ period, with 2 teams working simultaneously at Matanzas and Pucatrihue, $1050 \mathrm{~km}$ apart.

Data recorded from the above experiments included the number of fronds larger than $2 \mathrm{~cm}$, frequency of infection and reproductive status of Mazzaella laminarioides. Transplants were monitored monthly during 10 mo, beginning in May 1995. Data were analyzed by 1-way ANOVAs for comparisons between controls and treatments for each sampling date. Time was not included as a factor because our objective was to detect the moment when experimental units became either different or equal to controls.

Diseases and frond survivorship. To assess the effect of endophytic infections on the permanence of diseased fronds in the population, selected thalli of Mazzaella laminarioides were tagged with FTF-69 Fingerling tags (Floy Tag) at the narrow end of the stipe. Two treatments were included: (1) intact fronds and (2) trimmed fronds (i.e. cut with scissors). A total of 40 intact individual fronds infected by Pleurocapsa sp., 40 infected by Endophyton ramosum, and 40 uninfected (control) were tagged in May 1995. The experiment was repeated during September 1995 in the 2 localities. The experimental design for trimmed fronds was the same as for intact fronds, although in this case the number of tagged fronds was 20 for each condition (i.e. infected by E. ramosum, infected by Pleurocapsa, and uninfected controls). The experiment with trimmed fronds was done in May and repeated during September 1995. To control tagging quality, 120 tags were fastened to aluminum screws secured to the rocks and scattered within the experimental area. The results were compared at the end of each experimental period by using a 2 -percentage comparison test as described. by Sokal \& Rohlf (1981).
Two additional experiments were implemented to specifically test the hypothesis that the green patch disease modifies the biomechanical properties of the thallus, rendering it weaker and, therefore, more susceptible to being dislodged from the holdfast by wave action. First, a total of 15 fronds, heavily infected by Endophyton ramosum, were selected for breaking trials. An equal number of uninfected fronds was used as controls. Each frond was clamped at the lesion using plastic clamps with sponge-protected borders to avoid cuts in the thallus. The clamp grabbing the proximal. part of the thallus was fixed to a wooden bench, and the clamp grabbing the distal part of the thallus was hooked to a dynamometer with a lever (modified from Denny 1988). Distance between the 2 clamps was approximately $2 \mathrm{~cm}$. Prior to the trials, accuracy of the dynamometer was calibrated using known weights. To avoid tissue dehydration, fronds were continuously sprayed with seawater during the trials. Only breaks through the center of the lesion (i.e. more or less equidistant from each clamp) were considered valid and, in those cases, the cross-section area of the thallus at the breaking point was measured directly using a micrometric scale. Failure of the frond was assessed by manually pulling the free end of the dynamometer, at a speed of $0.06 \mathrm{~m} \mathrm{~s}^{-1}$ until the tissue fractured.

The second experiment was designed as a complement to the in vitro measurements of breaking stress. An area of $3 \times 0.5 \mathrm{~m}$ within the low-infection zone of Matanzas was selected for this purpose. The selected area is characterized by heavy wave impact; preliminary measurements (Correa, Buschmann, Beltrán \& Retamales unpubl.) show water velocity values ranging from 5.4 to $9.4 \mathrm{~m} \mathrm{~s}^{-1}$. Thirty fully diseased fronds and 30 healthy fronds, similar in size 10 to $12 \mathrm{~cm}$ in length) and attached to the rocks by their respective hold fasts, were chiseled off as in the transplant experiments. Fronds for this experiment were similar in size, were selected at the same time and from the same area as those used in the fracture experiment. Rock fragments containing the experimental individuals were glued to the rocky platform at the selected site using submarine epoxy cement (Stierling). To avoid the effect of unforeseen heterogeneity in the experimental site on frond dislodgment, rock fragments bearing diseased individuals were interspersed among fragments with healthy fronds

Diseases and herbivory. Herbivore and algal collections for laboratory feeding assays were made in Pucatrihue. In this locality, the gastropod Tegula atra Lesson and the amphipod Hyale hirtipalma Dana are among the most abundant herbivores (Jara \& Moreno 1984, Buschmann 1990), and for the feeding assays they were collected directly from the Mazzaella laminarioides stands. 
Amphipods and gastropods were collected $4 \mathrm{~d}$ prior to the feeding assays. The specimens were held in $5 \mathrm{l}$ plastic containers with aerated seawater and no food supply. Seawater used in the trials was taken from the collection site and changed daily.

Mazzaella laminarioides fronds used in the assays were collected $24 \mathrm{~h}$ prior to the trials, transported in cooled containers to the laboratory and sorted into 3 categories: fronds without disease symptoms, fronds heavily infected by Pleurocapsa sp. and fronds heavily infected by Endophyton ramosum. As a way to assess normality of the feeding behavior of grazers during the trials, the highly palatable Ulva rigida was included in the tests. Only vegetative, epiphyte-free tissue with no obvious herbivore damage was used throughout the trials.

The no-choice and multiple-choice feeding assays with Tegula atra were done in 51 plastic containers. Amphipod feeding experiments were carried out in $150 \mathrm{ml}$ plastic disposable cups, as described elsewhere (Buschmann \& Santelices 1987). In all cases, the experiments were done using a $12 \mathrm{~h}$ light: $12 \mathrm{~h}$ dark photoperiod, a photon flux density of $20 \mu \mathrm{mol} \mathrm{m} \mathrm{m}^{-2} \mathrm{~s}^{-1}$, a water temperature of 12 to $15^{\circ} \mathrm{C}$ and a salinity of $32 \mathrm{ppt}$.

In the multiple-choice assays with Tegula atra, 5 specimens $\left(22.3 \pm 2.5 \mathrm{~g}\right.$ fresh weight container $\left.{ }^{-1}\right)$ were added to each of 5 containers. In the equivalent experiments with Hyale hirtipalma, 5 individuals (76.3 \pm $14.4 \mathrm{mg}$ fresh weight $\mathrm{cup}^{-1}$ ) were added to each of 8 cups. To assess changes in seaweed biomass not due to grazing activity, controls containing seaweeds without grazers were included in the experimental design. The number of control replicates was the same as for treatment replicates ( 5 for $T$. atra and 8 for $H$. hirtipalma), as indicated by Roa (1992), and containers were arranged randomly in a 2 -dimensional matrix throughout the trials. In the experiments with T. atra, $700 \pm 50 \mathrm{mg}$ of each of the 4 algal items were added to each container and the animals were allowed to graze for $112 \mathrm{~h}$. In the amphipod experiments, $160 \pm 3 \mathrm{mg}$ of each algal item were added to the cups and the animals were allowed to graze for $120 \mathrm{~h}$. The multiple-choice experimental design violates the assumption of independence among treatments and, therefore, it is incorrect to use ANOVA for univariate analysis (Peterson \& Renaud 1989, Holmlund et al. 1992). More recently, Roa (1992) applied a random subtraction of the controls to eliminate autogenic changes from the data, without underestimating the error variance. The analysis can proceed by applying a 1 -sample Hotelling's $\mathrm{T}^{2}$ test using SYSTAT (Wilkinson et al. 1992).

No-choice feeding experiments were similar to the multiple-choice trials, although only $2.5 \pm 0.1 \mathrm{~g}$ of a single food item was offered to Tegula atra and $160.0 \pm$
$0.01 \mathrm{mg}$ to Hyale hirtipalma. The experimental design included 5 replicates for each treatment (i.e. food items offered) in the gastropod experiment, and 10 replicates in the amphipod trial. Gastropod and amphipod trials included 5 and 10 additional replicates, respectively, as controls without grazers. At the end of the experiments, the remaining algal tissue was blotted and weighed to allow calculation of tissue loss due to grazing and biomass increase due to growth. Difference in wet weight between control and treatment was used to estimate net loss of Ulva rigida and infected and uninfected Mazzaella laminarioides. A single factor ANOVA was used after checking for homoscedasticity (Bartlett's test) and normality (visual inspection of frequency distribution data). Prior to that, data were logarithmically transformed. Pairwise combinations were tested using the Tukey's test.

\section{RESULTS}

\section{Disease expression}

In the high-infection zone at Matanzas (Fig. 2a), control transplants showed a clear decrease in diseased fronds toward late winter-early spring, from $100 \%$ in May to less than $30 \%$ in October. The frequency of diseased fronds began increasing in November and reached $100 \%$ in February. Transplants of local Mazzaella laminarioides fronds from the low-infection zone began a steady increase of visually detectable diseased fronds from zero in May to almost $8 \%$ in August. In November, the frequency of diseased fronds showed a sharp increase with mean values not significantly different ( $F=0.66, p=0.42$ ) from the controls. From November, the frequency of diseased fronds in transplant units from the low-infection zone progressed almost identically to the control units. Mazzaella laminarioides from the low-infection zone of Pucatrihue responded, initially, in a similar fashion to their counterparts from Matanzas, with a clear increase in the frequency of diseased fronds in December. However, infection frequency reached a maximum value only slightly higher than $60 \%$ in February, and it was significantly different $(F=11.05, \mathrm{p}<0.01)$ from both controls and local fronds.

At the high-infection zone of Pucatrihue (Fig. 2b), control fronds followed a trend similar to that recorded in Matanzas. Frequency of diseased fronds in the controls decreased sharply in October, to fall below $40 \%$ during November-December. The frequency of diseased fronds in these controls then increased to reach $90 \%$ in February. Transplants of local Mazzaella laminarioides from the low-infection zone remained with the background level of macroscopically diseased 

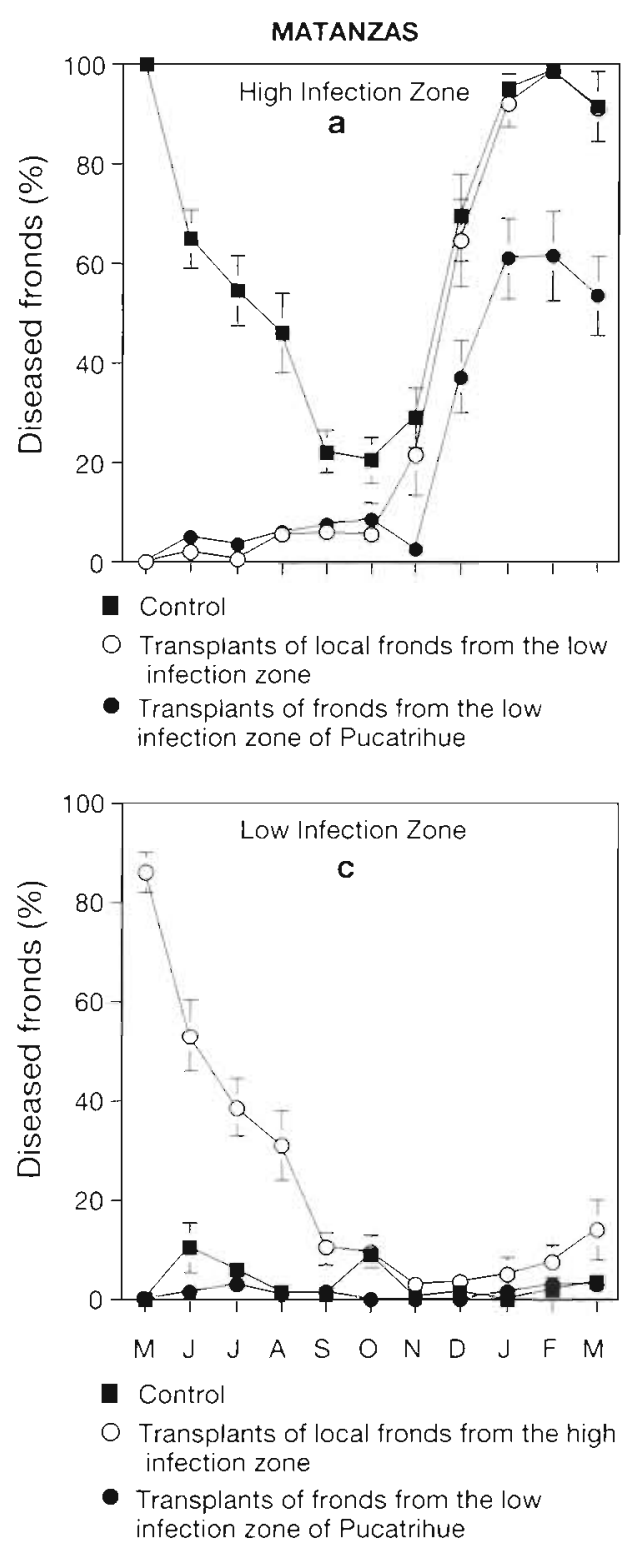

PUCATRIHUE

Transplants of fronds from the low infection zone

- Transplants of fronds from the low infection zone of Matanzas

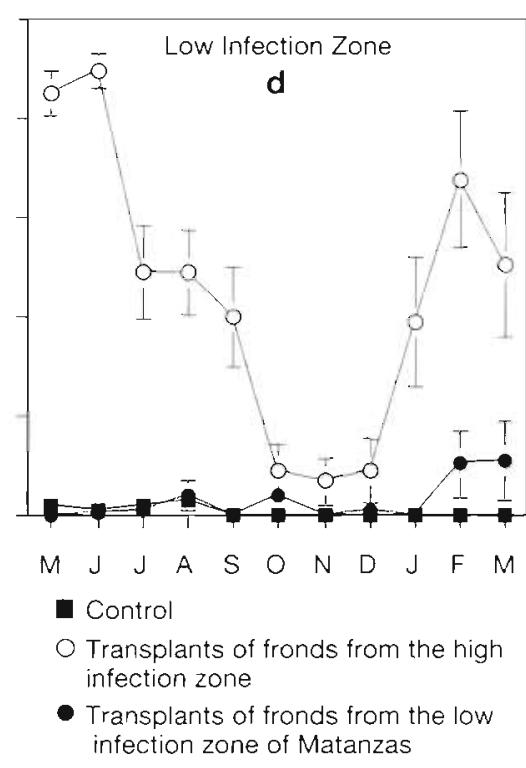

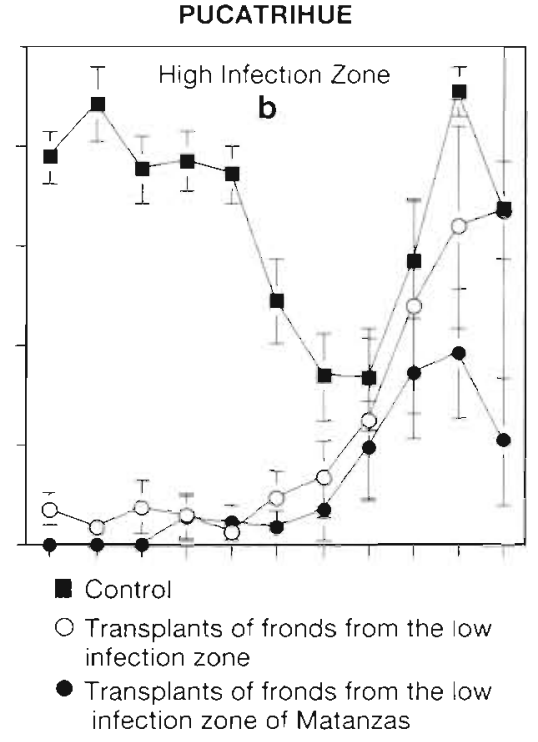

Fig. 2. Disease expression in Mazzaella laminarioides fronds (mean frequency $\pm 1 \mathrm{SE}$ ) at the high-infection zones in Matanzas (a) and Pucatrihue (b), and at the low-infection zones in Matanzas (c) and Pucatrihue (d) fronds until October, where the frequency of diseased fronds began to increase steadily. In November, these transplant units reached frequencies of diseased fronds not significantly different $(F=3.21, \mathrm{p}=0.08)$ from the controls, a situation which prevailed until the end of the experiment. The frequency of diseased fronds in the transplant units from the low-infection zone of Matanzas remained below 10\% until November. By December, frequency of diseased fronds in these transplants reached a mean value of $20 \%$, which was not significantly different $(F=1.99, \mathrm{p}=0.17$ ) from the controls. By March, however, in spite of the almost $40 \%$ recorded during January-February, the frequency of diseased fronds in these transplants dropped significantly $(F=19.79, \mathrm{p}<0.01)$, to $20 \%$, as compared to the $70 \%$ recorded in the controls.
At the low-infection zone of Matanzas (Fig. 2c), the frequency of diseased fronds in the controls remained low during most of the experiment, with the exception of June and October, when mean values of $10 \%$ were recorded. Transplants from the high-infection zone of Matanzas showed a rapid decline in the frequency of diseased fronds, from almost $90 \%$ in May to less than $5 \%$ in November, with no significant differences $(F=2.54$. $p=0.12$ ) from the controls in the latter case. By March, frequency of diseased fronds increased to a mean value of $18 \%$, although this was still statistically similar $(F=$ $2.56, p=0.12)$ to the controls. Transplants from the lowinfection zone of Pucatrihue displayed an almost total absence of visually detectable diseased fronds, showing no significant differences with the controls $(F=0.01$, $p=0.91$ ) throughout the experimental period. 
At the low-infection zone of Pucatrihue (Fig 2d), fronds in the controls remained almost free of symptoms throughout the experiment. Transplants from the high-infection zone of Pucatrihue showed a decline from almost $90 \%$ diseased fronds in May to less than $10 \%$ during October-December. Frequency values in November and December were statistically similar $(F=1.97, \mathrm{p}=0.17 ; F=2.34, \mathrm{p}=0.14$, respectively) to the controls. However, the infection prevalence in these transplants increased significantly ( $F=$ 7.89, $p<0.01$ ) from January to March. Transplants from Matanzas to Pucatrihue did not show significant differences $(F=2.17, \mathrm{p}=0.17)$ from the controls throughout the experiment. The increase in the frequency of infection observed in these transplants dur- ing February and March was not significantly different from the controls.

In general, a decrease in the percentage of diseased fronds was recorded (Fig. 2) in association with an actual decrease in the number of diseased fronds (Fig. 3). In some cases, the decrease in diseased fronds was accompanied by a simultaneous increase in the total number of fronds. On the other hand, when an increase in the percentage of diseased fronds was recorded (Fig. 2), it was associated with an increase in the total number of fronds (Fig. 3).

Representative individual fronds of Mazzaella laminarioide were collected at the end of the experimental period (Figs. 4 to 9). Fronds representing the transplants from the low-infection zone of Pucatrihue to the
MATANZAS

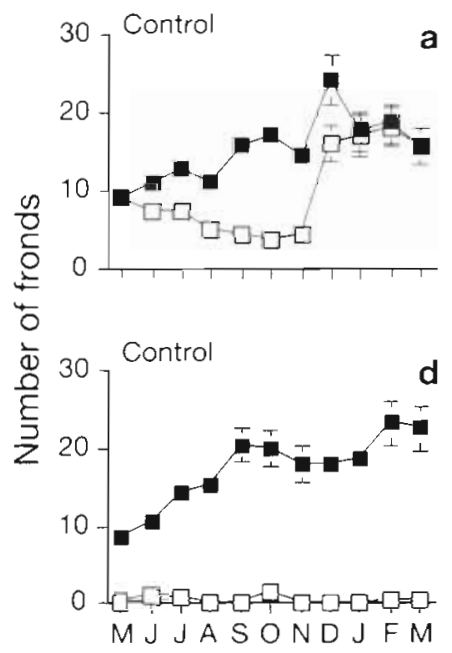

PUCATRIHUE

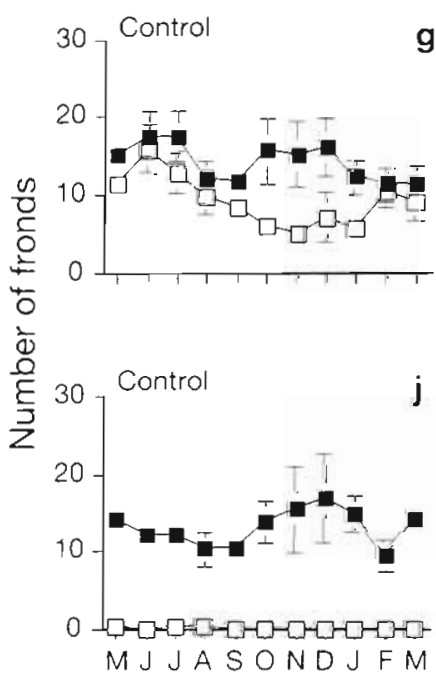

High Infection Zone

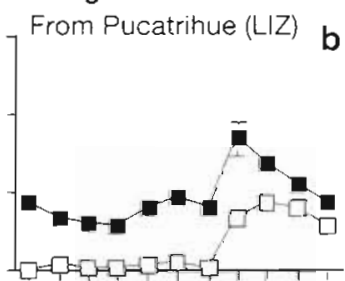

Low Infection Zone

From Pucatrihue (LIZ)

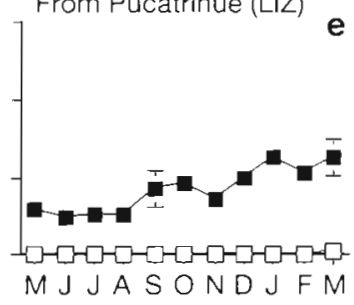

High Infection Zone

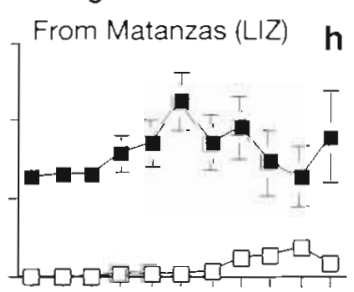

Low Infection Zone

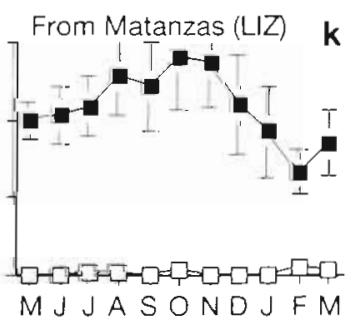

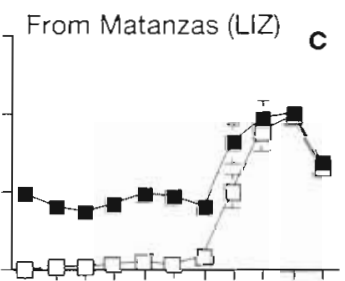

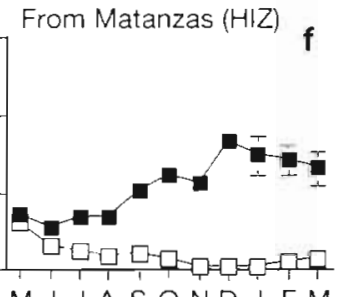

$M J J A S O N D J F M$

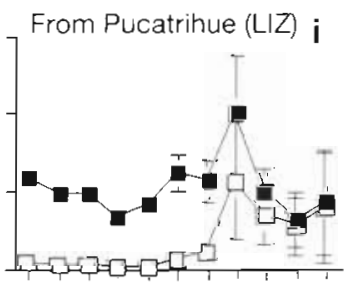

From Pucatrihue (HIZ) I

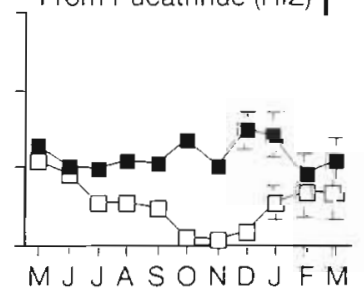

Total fronds $\square$ Diseased fronds

Fig. 3. Changes in Mazzaella laminarioides total frond numbers and diseased frond numbers during the transplant experiments in Matanzas (a to f) and Pucatrihue ( $g$ to l). Each point represents the mean ( $\pm 1 \mathrm{SE}$ ) for 15 transplant units. (a) Control for the Matanzas high-infection zone responses, (b) transplants from low-infection Pucatrihue to high-infection Matanzas, (c) transplants from low-infection Matanzas to high-infection Matanzas, (d) control for low-infection zone responses, (e) transplants from low-infection Pucatrihue to low-infection Matanzas, and (f) transplants from high-infection Matanzas to low-infection Matanzas. (g) Control for the Pucatrahue high-infection zone responses, (h) transplants from low-infection Matanzas to highinfection Pucatrihue, (i) transplants from low-infection Pucatrihue to high-infection Pucatrihue, (j) control for lowinfection zone responses, (k) transplants from low-infection Matanzas to low-infection Pucatrihue, (1) and transplants from high-infection pucatrihue to low-infection Pucatrihue 

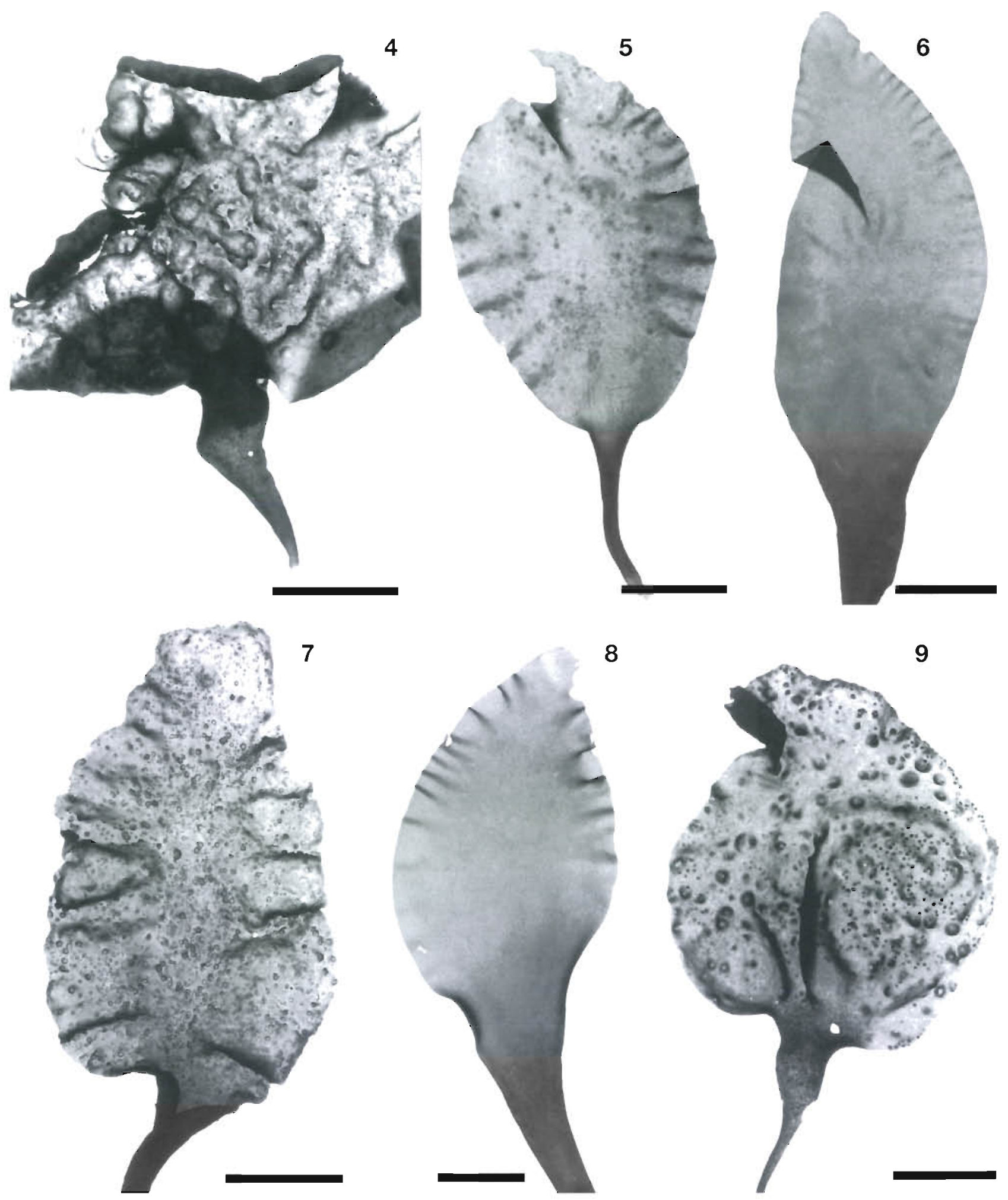

Figs. 4 to 9. Mazzaella laminariordes. Representative examples of the morphological changes occurring in fronds during the transplant experiments as recorded at the end of the experimental period. Fig. 4 Grossly deformed frond in transplant from Pucatrihue (low-infection zone) to Matanzas (high-infection zone). Scale bar $=2 \mathrm{~cm}$. Fig. S. Morphologically normal frond from Pucatrihue (low-infection zone) to Matanzas (low-infection zone). Circular, dark spots on the frond correspond to mature cystocarps. Scale bar $=2 \mathrm{~cm}$. Fig. 6 . Normal frond from the control units at the low-infection zone of Matanzas. Scale bar $=2 \mathrm{~cm}$. Fig. 7. Heavily diseased frond from the control units at the high-intection zone of Matanzas. Scale bar $=1.5 \mathrm{~cm}$. Fig. 8 . Morphologically normal frond from a transplant unit originating at the high-infection zone of Matanzas and transplanted to the lowinfection zone of the same locality. Scale bar $=1.5 \mathrm{~cm}$. Fig. 9. Deformed frond from a transplant unit originating at the lowinfection zone of Matanzas and transplanted to the high-infection zone of the same locality. Scale bar $=2 \mathrm{~cm}$ 


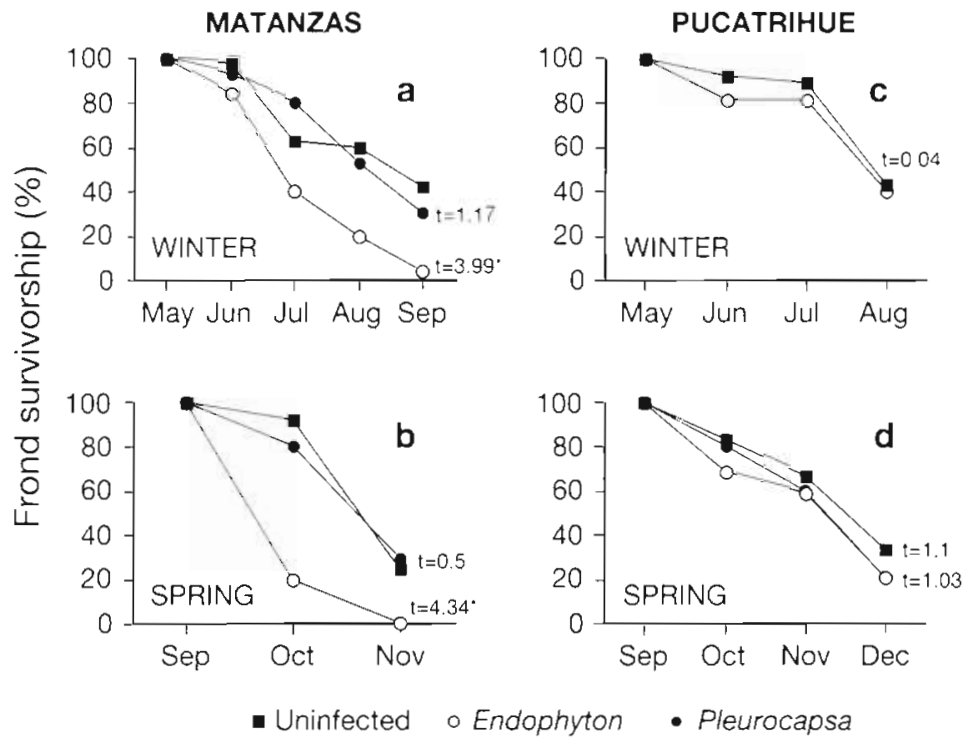

Fig. 10. Survivorship of whole Mazzaella laminarioides fronds in tagging experiments in Matanzas during (a) winter and (b) spring, and in Pucatrihue during (c) winter and (d) spring

vivorship in both winter (Fig. 10a; $t=3.99, \mathrm{p}$ $<0.05$ ) and spring (Fig. 10b; $t=4.34$, $p<0.05)$. Survivorship of fronds infected by Pleurocapsa sp. (Fig. 10a, b) was not significantly affected in winter $(t=1.17, \mathrm{p}>0.05)$ or spring $(t=0.50, p>0.05)$. In Pucatrihue, on the other hand, a negative effect of infection by $E$. ramosum on frond survivorship was not observed in winter $(t=0.04, \mathrm{p}>0.05)$ or spring $(t=1.03, p>0.05)$. The effect of Pleurocapsa sp. (Fig. 10c, d) on frond survivorship was not significant in spring $(t=1.1, p>$ 0.05 ) information for winter is not available. high-infection zone of Matanzas showed the typical symptoms of the deformative disease (Fig, 4). This contrasted with fronds of normal appearance, characteristic of the transplants from the low-infection zone of Pucatrihue to the low-infection zone of Matanzas (Fig. 5), and with those of the Matanzas controls for the low-infection zone (Fig. 6). Transplants located at the high-infection zone of Matanzas, including controls (Fig. 7) and units from the low infection zone (Fig. 9), usually showed a high number of severely diseased fronds. On the other hand, most fronds (>80\%; Fig 2c) in the transplants from the high-infection zone of Matanzas to the lowinfection zone of the same locality did not reveal disease symptoms (Fig. 8).

\section{Diseases and frond survivorship}

Tagging experiments in Matanzas showed that infection by Endophyton ramosum had a significant and negative effect on frond sur-

Fig. 11 Survivorship during tagging experiments of trimmed Mazzaella laminariondes fronds infected by Endophyton ramosum durng (a) winter and (b) spring and by pleurocapsa sp. during (c) winter and (d) spring in Matanzas, and survivorship of fronds infected by E. ramosum dunng (e) spring and by Pleurocapsa sp. during (f) winter and (g) spring in Pucatrihue

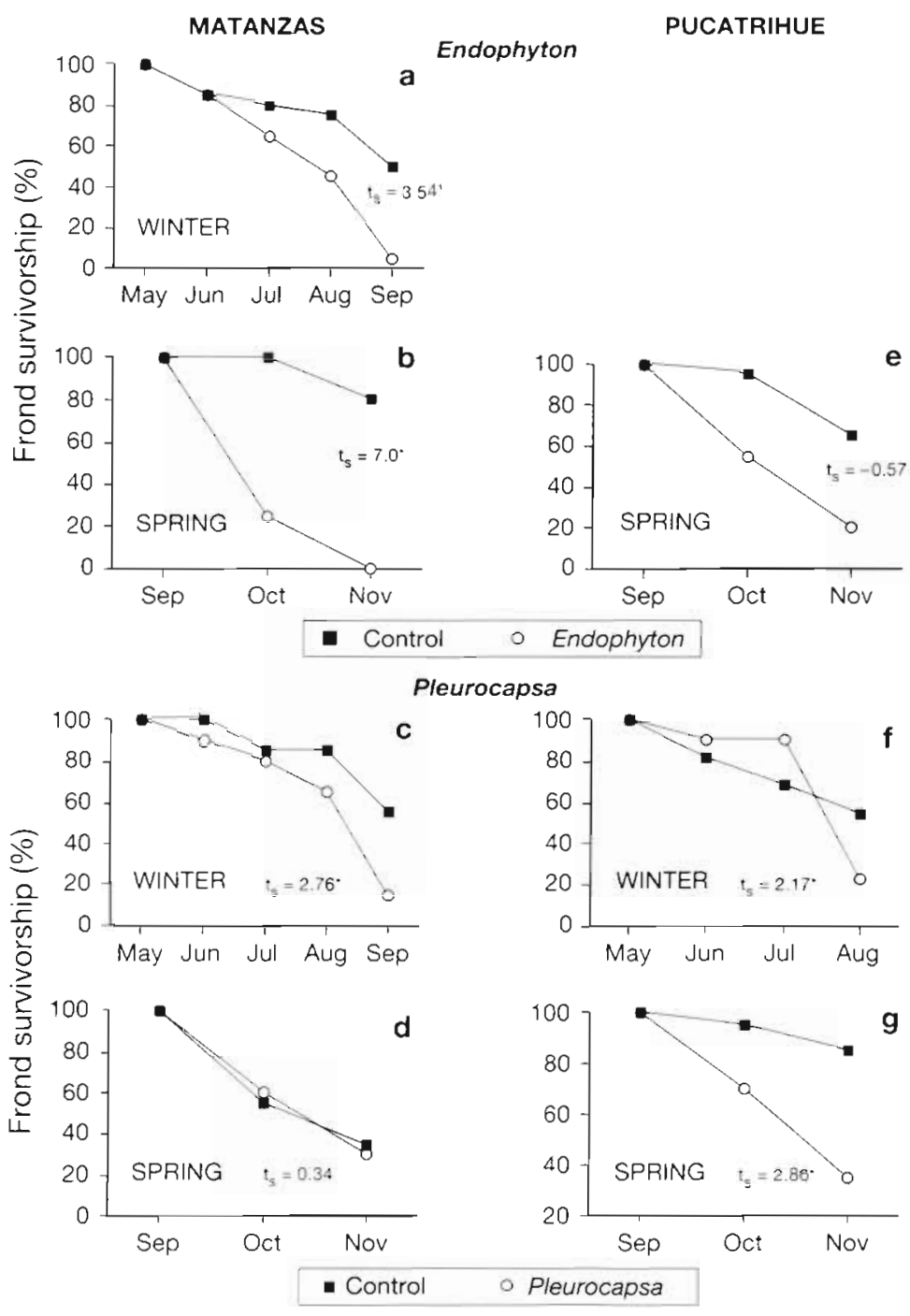




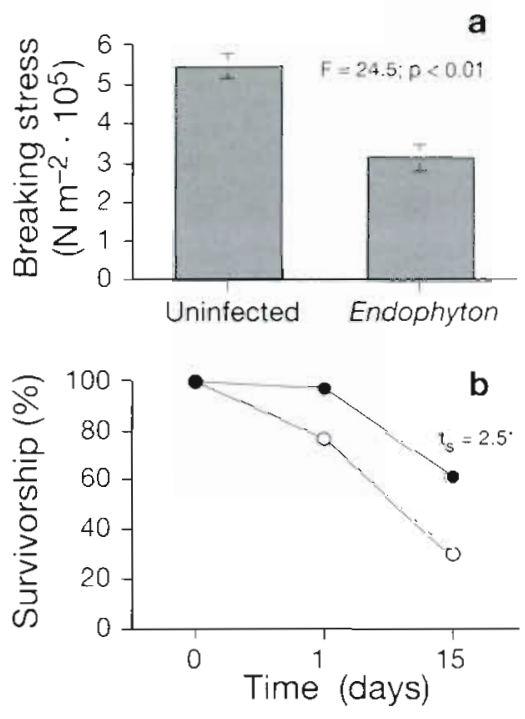

- Uninfected OEndophyton

Fig. 12. Effects of severe infection by Endophyton ramosum on the biomechanical properties of the tissues of Mazzaella laminarioides. Fronds allocated to each experiment came from a single pool and were randomly assigned to either

(a) breaking stress or (b) survivorship trials

Presence of trimmed fronds infected by Endophyton ramosum in Matanzas decreased significantly in the population during winter (Fig. 11a $t=3.54, \mathrm{p}<0.05$ ) and spring (Fig $11 \mathrm{~b} ; t=7, \mathrm{p}<0.05$ ). Similar responses were found in fronds infected by Pleurocapsa sp. during winter (Fig. $11 \mathrm{c}_{;} t=2.76, \mathrm{p}<0.05$ ), but not during spring (Fig. 11d $t=0.34, \mathrm{p}>0.05$ ). As in Matanzas, the effect of infections on survivorship of trimmed fronds in Pucatrihue varied according to the pathogen and season (Fig. 11e to g). Fronds infected by E. ramosum showed survival rates significantly lower $(t=-0.57, \mathrm{p}<$ 0.05 ) than controls during spring (Fig. 11e). Infections by Pleurocapsa sp., on the other hand, resulted in a significantly $(t=2.86, p<0.05)$ faster removal of diseased individuals during spring (Fig. 11.g), but not during winter (Fig. 11f; $t=2.17, \mathrm{p}>0.05$ )

In vitro breaking trials showed that tissues affected by the green patch disease broke consistently at the lesion, and required less force to be broken than healthy thalli (Fig. 12a; $F=24.5, p<0.01$ ). Consistent with the above results, the transplant experiment associated with the breaking trials showed that diseased fronds were lost significantly faster (Fig. 12b; $t=2.5$, $p>0.05$ ) than healthy controls.

\section{Diseases and herbivory}

When all 4 food items were offered simultaneously, Tegula atra consumed significantly more tissue from Ulva and Mazzaella laminarioides affected by the deformative disease than $M$. laminarioides affected by the green patch disease and non-infected controls
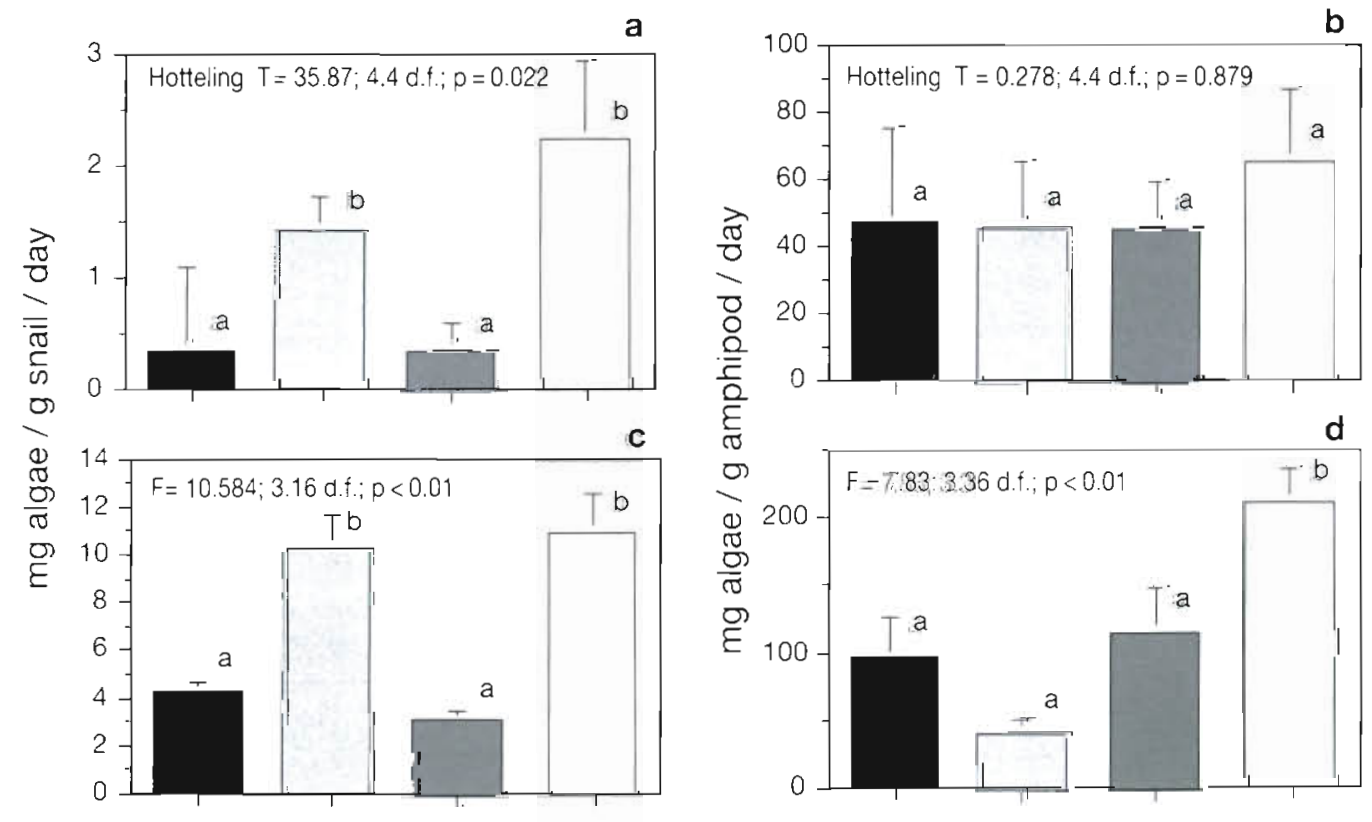

Healthy $M$. laminarioides $\square M$. laminarioides - D.D

M. laminarioides - G.P.D. $\square$ Ulva rigida

Fig. 13. Effects of the green patch disease and deformative disease on consumption of algal tissue by gastropod and crustacean grazers. Tissue consumption (mean $+1 \mathrm{SE}$ ) by (a) Tegula atra and (b) / I jale hirtipalma in multiple-choice experiments. Tissue consumption (mean $+1 \mathrm{SE}$ ) by (c) T. atra and (d) H. hirtipalma in no-choice experiments. D.D.: deformative disease; G.P.D.: green patch disease. Different letters over the bars denote significant $(p<0.05)$ differences 
(Fig. 13a) The amphipod Hyale hirtipalma, on the other hand, did not discriminate among the different food items when they were offered simultaneously (Fig. 13b). In the no-choice experiments, $T$. atra repeated the preference pattern shown in the multiplechoice trials, removing significantly higher biomass from $M$. laminarioides affected by the deformative disease and from Ulva rigida (Fig. 13c). Differences between biomass removed from non-infected $M$. Jaminarioides and tissue affected by the green patch disease were not significant (Fig. 13c). No-choice trials with $H$. hirtipalma showed that the amphipod consumed diseased (deformative and green patch diseases) and non-diseased $M$. laminarioides at the same rate, whereas $U$. rigida was consumed at a greater rate (Fig. 13d).

\section{DISCUSSION}

This study demonstrates that the expression of the deformative disease in Mazzaella laminarioides is strongly influenced by the location of the fronds on the shore. This is clearly indicated, for example, by the reciprocal transplants within a locality, where transplanted units developed frequencies of infection almost identical to the controls. That is, transplants to the highinfection zones became highly infected whereas those to the low-infection zones rapidly lost their diseased fronds and new, non-infected thalli developed, resulting in transplant units with low infection frequencies. A deviation from this pattern occurred in the lowinfection zone of Pucatrihue, where the low frequency of diseased fronds only lasted 3 mo and was followed by a re-appearance of up to $65 \%$ diseased thalli. However, the pattern of disease re-appearance displayed by the transplants at the low-infection zone of Pucatrihue differs from the seasonal fluctuation displayed by their counterparts maintained as controls at the high-infection zone. Whereas in the latter case the frequency of diseased fronds remained high until September, the lowest values were about $35 \%$ and the re-appearance values were higher than $90 \%$; at the low-infection zone the decline in diseased fronds began almost immediately and resulted in frequencies lower than 10\% Furthermore, the frequency of diseased fronds (ca 65\% in February, ca 50\% in March) in. these transplants toward the end of the experiment was lower than the values of the same transplants at the beginning of the experiment and also lower than their counterparts at the high-infection zone at the same time of the year Although the cause of the increase in the frequency of diseased fronds at the lowinfection zone of Pucatrihue remains to be elucidated, we hypothesize that the low values observed from
October to December were still high enough to trigger an outbreak during the following summer. We believe that, with time, a steady state will be reached, where the late-winter diseased survivors will occur at such low frequencies that the summer outbreak will no longer occur. This hypothesis seems to be supported by the progress of the disease in the local transplants from the high-infection zone to the low-infection zone of Matanzas. There, the rate of diseased fronds was almost zero in November and December and, consistent with our hypothesis, summer expression of the disease only affected $15 \%$ of the transplanted population, lower than the $100 \%$ reached at the same time of the year by their counterpart transplants maintained as controls at the high-infection zone of Matanzas.

The results of the cross-locality transplants also support the hypothesis that disease expression seems strongly influenced by the spatial location of the host on the shore. In Matanzas for example, transplant units from the low-infection zone of Pucatrihue displayed either high or low disease frequencies at the end of the experiment, depending on whether they were transplanted to the high-or low-infection zones. A similar pattern of response was detected in Pucatrihue. Interestingly, foreign transplant units at the high-infection zones of both Pucatrihue and Matanzas did not reach the levels of infection developed in the within-locality transplants. This evidence suggests that at least part of the expression of the deformative disease in Mazzaella laminarioides may be controlled endogenously. Although little is known regarding host specificity of the pathogen Pleurocapsa sp., Correa et al. (1993) indicated that only $M$. laminarioides appeared affected in the field. Furthermore, no differences in susceptibility to infection in the 2 life history phases of $M$. laminarioides have been reported for wild populations of the host (Correa et al. 1993, Correa \& Sánchez 1996). Still, subtle differences may have evolved between the populations of both hosts and pathogens at Matanzas and Pucatrihue in such a way that the disease is no longer transmitted to foreign fronds at the rate it is transmitted to local thalli (i.e incomplete hostpathogen compatibility). Differences in infectivity, and the subsequent development of the symptoms, among endophytic isolates from different origins was recently reported by Sánchez et al. (1996) for the Endophyton ramosum-M. laminarioides association. Unfortunately, very little is known about the molecular basis of virulence and susceptibility in algae, although in higher plants and their fungal and bacterial pathogens it is clear that a genetic match between host and pathogen must exist if full disease expression is to develop (see Agrios 1988)

Our results support, in general, the hypothesis that diseases may affect survivorship, although caution is 
needed due to the apparent locality-dependence of this phenomenon. For example, whereas in Matanzas a clear negative effect on frond survivorship was apparent regardless of season and the pathogen involved, in Pucatrihue both diseased and healthy fronds were lost at the same rates. So far, we have not identified the reason for this apparent variability associated with locality, and further research is needed in this area. The only additional information available on the effects of diseases on host survivorship in wild populations appeared recently (Correa \& Sánchez 1996) and also involved the Mazzaella laminarioides population at Matanzas. In that report, a decreased survivorship of diseased fronds was recorded, showing consistency with our present results for that locality. Trimming of fronds did not modify the general survivorship responses observed in whole fronds from Matanzas, although it did accelerate mortality of diseased fronds in Pucatrihue. Bearing in mind the variability mentioned above, it seems clear that the hypothesis that Endophyton ramosum acts as a weakening agent, modifying some biomechanical properties of the infected tissues, is supported by our results. The force needed to fracture healthy thalli is almost twice the force required to break diseased fronds. This further explains the loss of $70 \%$ of diseased fronds exposed to heavy wave impact during 15 d compared to loss of only $35 \%$ of healthy individuals during the same period of time. The negative effect of pathogens on biomechanical properties of an algal host was first tested in the Chondrus crispus-Acrochaete operculata association by Correa \& McLachlan (1992), who demonstrated in in vitro trials that infected tissues became debilitated. Subsequently (Correa \& McLachlan 1994), it was shown that such a weakening was caused by a direct host cell destruction and that such a primary destruction was enhanced by secondary damage induced by bacterial invasion. Observations on damage at cellular and tissue levels similar to those reported in $C$. crispus were described by Correa et al. (1994) in M. laminarioides infected by E. ramosum. As a consequence, these authors suggested that $M$. laminarioides, like $C$. crispus, could be biomechanically affected as a result of the green patch disease, a phenomenon that has been confirmed in the present study.

Selective grazing of diseased tissue can represent an additional side-effect on infected hosts. This was clearly the case in fronds affected by the deformative disease which were grazed preferentially by Tegula atra. Selective grazing, however, varies according to the grazer and to the disease affecting the host. The gastropod $T$. atra was not attracted by host tissue affected by the green patch disease, whereas Hyale hirtipalma was not attracted to diseased hosts at all. These findings somewhat deviate from the results expected based upon the Acrochaete operculataChondrus crispus pathosystem, where invasion by the green alga triggered significant changes in herbivory involving an enhanced consumption by the common isopod Idotea baltica (Correa \& McLachlan 1992). Even though selective grazing does not seem to be of importance for Mazzaella laminarioides affected by the green patch disease, tissue erosion at the lesion caused by grazing may still represent the beginning of material failure, potentially leading to fracture (see Denny 1988, Correa \& McLachlan 1992). Furthermore. it has to be considered that amphipods preferentially consume cystocarpic $M$. laminarioides (Buschmann \& Santelices 1987, Buschmann 1991). This, together with the observations that fertile fronds tend to be more infected by the endophytes (Correa et al. 1997), suggests that interactions between grazers and endophytism are complex and require further attention.

In conclusion, this study provides evidence which supports the hypothesis that the patchy pattern of expression of the deformative disease in Mazzaella laminarioides depends strongly upon exogenous factors, still not identified, but likely associated with microenviromental conditions resulting from withinlocality heterogeneity. However, our data from between-locality transplants also suggest that at least part of the disease expression may be determined endogenously. Furthermore, information gathered in this study helps to elucidate the potential role of 2 important infectious diseases on host mortality and grazing enhancement. Regarding these 2 last points, variability in the observed responses depended upon locality, disease and species of herbivore.

Acknowledgements. This study was supported by research grant FONDECYT 1940647 to J.A.C. and A.H.B. We are grateful for field and laboratory' help provided by Jaime Montenegro, Jorge Lucic, César Pinto, Pablo Sánchez, Diego Aedo and Marcelo Bobadilla.

\section{LITERATURE CITED}

Agrios GN (1988) Plant pathology, 3rd edn. Academic Press, Inc, San Diego

Buschmann AH (1990) Intertidal macroalgae a refuge and food for Amphipoda in central Chile. Aquat Bot 36: $237-24.5$

Buschmann AH (1991) Amphıpod food preference and Iridaea spp. (Rhodophyta) spore release and dispersal. J Mar Bıol Assoc UK 71:891-897

Buschmann AH, Santelices B (1987) Micrograzers and spore release in Iridaea laminarioides Bory (Rhodophyta: Gigartinales). J Exp Mar Biol Ecol 108:171-179

Corred JA (1997) Algal diseases. In: Round F, Chapman DJ (eds) Progress in phycological research. Biopress Ltd, Bristol (in press)

(orrea JA, Buschmann A, Retamales C, Beltrán J (1997) 
Infectious diseases of Mazzaella laminarioides (Rhodophyta): changes in infection prevalence and disease expression associated with season, locality and withın-site location. J Phycol 33 (in press)

Correa JA, Flores V (1995) Whitening, thallus decay and fragmentation in Gracilaria chllensis associated with an endophytic amoeba. J Appl Phycol 7:421-425

Correa JA, Flores V, Garrido J (1994) Green patch disease in Iridaea laminarioides (Rhodophyta) caused by Endophyton sp. (Chlorophyta). Dis Aquat Org 19:203-213

Correa JA, Flores V. Sánchez P (1993) Deformative disease in Iridaea laminarioides (Rhodophyta): gall development associated with an endophytic cyanobactenum. J Phycol 29:853-860

Correa JA, McLachlan JL (1992) Endophytic algae of Chondrus crispus (Rhodophyta). IV Effects on the host following infections by Acrochaete operculata and A. heteroClada (Chlorophyta). Mar Ecol Prog Ser 81:73-87

Correa JA, McLachlan JL (1994) Endophytic algae of Chondrus crispus (Rhodophyta). V. Fine structure of the infection by Acrochaete operculata (Chlorophyta). Eur J Phycol 29:33-47

Correa JA, Sánchez PA (1996) Ecological aspects of algal infectious diseases. Hydrobiologia 326/327:89-95

Denny MW (1988) Biology and the mechanics of the waveswept environment. Princeton University Press, Princeton

This article was submitted to the editor
Holmlund MB, Peterson CH, Hay ME (1992) Does algal morphology affect amphipod susceptibility to fish predation? J Exp Mar Biol Ecol 139:65-83

Jara F, Moreno CA (1984) Herbivory and structure in a midlittoral rocky community: case in southern Chile. Ecology $65: 28-38$

Peterson CH, Renaud PE (1989) Analysis of feeding preference experiments. Oecologia 80:82-86

Roa R (1992) Design and analysis of multiple-choice feedingpreference experiments. Oecologia 89:509-515

Sánchez P, Correa JA, Garcia-Reina G (1996) Host-specificity of Endophyton ramosum (Chlorophyta), the causative agent of the green patch disease in Mazzaella laminarioides (Rhodophyta). Eur J Phycol 31:173-179

Santelices B (1991) Littoral and sublittoral communities of continental Chile. In: Mathieson AC, Nienhuis PH (eds) Ecosystems of the world (24). Intertidal and littoral ecosystems. Elsevier Science Publishers, New York, p $347-369$

Sokal RR, Rohlf FJ (1981) Biometry, 2nd edn. WH Freeman \& Co, New York

Weinberger F, Friedlander M. Gunkel W (1994) A bacterial facultative parasite of Graclana conferta. Dis Aquat Org 18:135-141

Wilkinson L, Hill MA, Vang E (1992) SYSTAT: statıstic, Version 5.2 edn. SYSTAT Inc, Evanston, IL

Manuscript received: March 18, 1997

Revised version accepted: May 22, 1997 